\title{
Quantifying the Effect of Social Presence on Online Social Conformity
}

\author{
SENURI WIJENAYAKE, The University of Melbourne, Australia \\ NIELS VAN BERKEL, Aalborg University, Denmark \\ VASSILIS KOSTAKOS, The University of Melbourne, Australia \\ JORGE GONCALVES, The University of Melbourne, Australia
}

\begin{abstract}
Social conformity occurs when individuals in group settings change their personal opinion to be in agreement with the majority's position. While recent literature frequently reports on conformity in online group settings, the causes for online conformity are yet to be fully understood. This study aims to understand how social presence i.e., the sense of being connected to others via mediated communication, influences conformity among individuals placed in online groups while answering subjective and objective questions. Acknowledging its multifaceted nature, we investigate three aspects of online social presence: user representation (generic vs. user-specific avatars), interactivity (discussion vs. no discussion), and response visibility (public vs. private). Our results show an overall conformity rate of $30 \%$ and main effects from task objectivity, group size difference between the majority and the minority, and self-confidence on personal answer. Furthermore, we observe an interaction effect between interactivity and response visibility, such that conformity is highest in the presence of peer discussion and public responses, and lowest when these two elements are absent. We conclude with a discussion on the implications of our findings in designing online group settings, accounting for the effects of social presence on conformity.
\end{abstract}

CCS Concepts: • Human-centered computing $\rightarrow$ Empirical studies in collaborative and social computing; Empirical studies in HCI.

Additional Key Words and Phrases: Online Social Conformity; Online Social Presence; Interactivity; Response Visibility; User Representation; Majority Size; Task Objectivity; Self-confidence

\section{ACM Reference Format:}

Senuri Wijenayake, Niels van Berkel, Vassilis Kostakos, and Jorge Goncalves. 2021. Quantifying the Effect of Social Presence on Online Social Conformity. 1, 1 (October 2021), 22 pages. https://doi.org/10.1145/nnnnnnn. nnnnnnn

\section{INTRODUCTION}

Conformity is a widely observed social phenomenon where individuals adjust their personal opinions to be in line with the group's expectations in an attempt to be 'liked' within the group (normative influence) or to be considered 'right' (informational influence) $[2,16]$. While conformity was initially studied in face-to-face settings, understanding its mechanisms in online settings is becoming increasingly important, primarily given that a growing range of societal interactions are now mediated through online technologies $[10,51]$.

Moreover, there is an increasing interest in online social conformity in the recent $\mathrm{HCI} / \mathrm{CSCW}$ literature. Individuals are seen to conform to contradicting opinions and norms set by majorities when

Authors' addresses: Senuri Wijenayake, swijenayake@student.unimelb.edu.au, The University of Melbourne, Parkville, VIC, 3010, Australia; Niels van Berkel, nielsvanberkel@cs.aau.dk, Aalborg University, Fredrik Bajers Vej 5, Aalborg, 9100, Denmark; Vassilis Kostakos, vassilis.kostakos@unimelb.edu.au, The University of Melbourne, Parkville, VIC, 3010, Australia; Jorge Goncalves, jorge.goncalves@unimelb.edu.au, The University of Melbourne, Parkville, VIC, 3010, Australia.

Permission to make digital or hard copies of all or part of this work for personal or classroom use is granted without fee provided that copies are not made or distributed for profit or commercial advantage and that copies bear this notice and the full citation on the first page. Copyrights for components of this work owned by others than ACM must be honored. Abstracting with credit is permitted. To copy otherwise, or republish, to post on servers or to redistribute to lists, requires prior specific permission and/or a fee. Request permissions from permissions@acm.org.

(C) 2021 Association for Computing Machinery.

XXXX-XXXX/2021/10-ART \$15.00

https://doi.org/10.1145/nnnnnnn.nnnnnnn

, Vol. 1, No. 1, Article . Publication date: October 2021. 
discussing political and social issues on social media [43,44], when completing online quizzes $[6,67]$ and visual judgement tasks [29], and when commenting on online news websites [60]. However, limited research has been undertaken to understand what triggers conformity in online settings. Furthermore, as online groups are inherently dissimilar to face-to-face groups due to reduced social contextual cues and the lack of physicality in online communication [45], previous work on wellunderstood determinants of face-to-face conformity (such as majority group size [3, 30,54], task objectivity (objective or subjective nature of the task) $[2,7,17]$, and self-confidence $[11,61]$ ), may not be of relevance in online group settings. Thus, further work is required to systematically investigate whether and how aforementioned determinants of face-to-face conformity manifest in online group settings, as an initial step towards fully understanding online social conformity.

Furthermore, as conformity is a form of social influence, the effect of social presence i.e., the awareness of and the sense of being connected to others via mediated communication [58], on online conformity behaviour has been an interest in recent literature [33, 37, 47]. However, the aforementioned studies limit their focus to only one aspect of online social presence (such as user representation [47], level of interactivity [33] or response visibility [37]), where as in realistic online settings these aspects are more likely to manifest together (e.g., online discussions usually involve certain user representations). Thus, in this study, we contribute to the existing literature by investigating the compound effects of three aspects of online social presence - user representation (generic vs. user-specific avatars), interactivity (discussion vs. no discussion) and response visibility (public vs. private responses) - on online conformity behaviour. Moreover, we investigate the effect of social presence in both objective and subjective quiz tasks, as individuals are challenged by majorities of different group sizes, while also accounting for their level of self-confidence to provide a wider understanding of online conformity determinants in comparison to existing work.

Our results reveal main effects from task objectivity, group size difference (difference between the majority group size and the minority group size), and initial self-confidence of the participant. We observe higher conformity in objective questions than in subjective questions, contradicting the findings of offline social conformity [7], likely due to the inherently lower social presence in online settings. We also note that larger majorities are more influential than smaller ones as previously suggested by both offline [3,20,54] and online [42, 65, 67] conformity literature. Moreover, participants are more likely to conform to the majority when they are less confident or uncertain of their initial answers, expanding the implications of existing literature on self-confidence and conformity in face-to-face groups $[11,61]$ to online settings.

While we do not observe main effects for the three aspects of social presence manipulated in this study, we note a statistically significant interaction effect between interactivity and response visibility. Conformity peaks in the presence of peer discussion and public responses, and remains at nominal levels in their absence. Thus, our findings imply that in addition to well-known conformity determinants such as majority size, task objectivity, and self-confidence, the level of social presence facilitated in an online setting plays a major role in users' conformity behaviour, making them especially susceptible to normative influences. We further discuss how this may be desired in settings where encouraging group norms is important (e.g., online support groups $[56,57])$, while it may have detrimental effects when conformity is not desired (e.g., online learning environments [6]). Thus, our findings should be taken into consideration when designing for online group settings in order to promote positive interactions.

\section{RELATED WORK}

In 1955, Asch set out to investigate the effect of peer opinions on individual judgements in face-toface group settings [3]. He employed a simple 'line matching' experiment, where for a significant number of responses (36.8\%) participants conformed to a clearly incorrect yet unanimous majority, establishing the susceptibility of individuals in group settings to conformity influences. Subsequently, Deutsch and Gerard [16] replicated Asch's experiment to reveal two motives behind group conformity: normative and informational influences. They described that individuals conform to the majority 
either with the intention of agreeing to their positive expectations (normative influence) or because they perceive information obtained from others to be more accurate evidence of a given situation than their own knowledge (informational influence). The notion of normative influence was further explained in $[14,38]$ as the inclination to ensure one's membership in a group, and seeking direction from the majority in uncertain situations as a manifestation of informational influences $[13,38]$.

Even though seminal social conformity literature is based on offline groups, owing to the recent movement of human societal interactions towards online platforms (e.g., social networks, learning platforms, discussion forums, support groups) [10, 21, 40, 51, 63], a growing number of HCI/CSCW researchers have sought to investigate the manifestation and implications of social conformity on online group settings as described below.

\subsection{Online Social Conformity}

Recent literature suggest that social conformity has diverse manifestations in a wide variety of online settings [6, 29, 43, 44, 56, 60, 69]. For instance, a study by Zhu et al. [69] investigated how individuals adjust their online choices when challenged by recommendations from other users. The study required participants to chose between two options, with and without the knowledge of others' preferences. They observed that an individual's choices in an online setting can be significantly swayed by others' opposing recommendations. Similarly, Maruyama et al. [43] note that people tend to adopt the majority's opinions when discussing social issues on online social networks, even when they are unaware of the users who are posting the content. Another study highlighted that users who were actively involved in Twitter during a televised political debate were more inclined to adjust their voting choice to support the majority sentiment on Twitter, further establishing the presence of social conformity in online settings [44].

Literature also provides evidence of the negative consequences of social conformity in certain online settings. For instance, Beran et al. [6] note that students who saw peer answers when completing an online quiz frequently conformed to the majority's answers, and obtained fewer correct answers compared to students who answered the quiz independently. A similar study by Hullman et al. [29] further emphasised the effect of social information (i.e., the notion that we tend to believe things more when we see others doing them) on the accuracy of a simple visual judgement task completed by Mechanical Turk users. They highlight that seeing biased and incorrect social information led to more errors, which could eventually nullify the expected benefits of collective intelligence.

However, online social conformity is not without its potential benefits. For example, Sukumaran et al. [60] highlight how normative influences can be used in online news websites to encourage high quality and 'thoughtful' contributions from its users. They note that when initial comments are of high quality, subsequent participants were also encouraged to contribute with similar effort. Similarly, previous work has shown that conforming to the acceptable conventions of behaviour and linguistic norms improved the sense of belonging and security within an online mental health support group, so that sensitive issues could be openly discussed [56].

The aforementioned literature indicates both positive and negative implications of online social conformity. We therefore argue that a thorough understanding of factors influencing this social phenomenon is critical to ensure that future online platforms facilitating social interactions are designed accounting for possible conformity effects. Therefore, we next summarise the existing literature on major determinants of social conformity as seen in both offline and online settings.

\subsection{Determinants of Social Conformity}

2.2.1 Majority Size. The majority group size (or the number of influential sources) on conformity behaviour has been popularly researched in offline groups and several theories have been put forward. For instance, Asch [3] observed that against a minority of one, the influential power of the majority increased until its third member, while adding a fourth member to the majority did not increase its conformity influence. Alternatively, Latané [34] proposed that while larger majorities have greater

, Vol. 1, No. 1, Article . Publication date: October 2021 
impact, the added impact is smaller for each additional group member. Similarly, the notion that larger majorities lead to higher conformity was further confirmed in subsequent studies $[30,46,54]$ However, the above studies considered unanimous majorities against a minority of one (participant) and did not account for the possibility of having larger minorities - which is typically the case in realistic online group settings.

Furthermore, a study by Lowry et al. [42] compared conformity behaviour across two group sizes (groups of three and six members) in both face-to-face and computer-mediated communication (CMC). They highlight that while conformity effects heightened in both conditions as the majority group size increased, the effect was lowered in the computer-mediated condition. Moreover, Walther et al. [65] investigated the effects of majority and minority group sizes on conformity in CMC group settings. Interestingly, their results show that the presence of minorities disturbing the unanimity of the group reduce the impact of the majority group size on conformity. However, while the significance of majority and minority group sizes on conformity behaviour has been suggested in the current literature, it is yet to be thoroughly investigated in an online setting.

2.2.2 Task Objectivity. Literature suggests that task objectivity can also play a significant impact on conformity behaviour. While conformity was initially tested in tasks of objective nature with an obvious correct answer [3,16], researchers were later interested in investigating how conformity manifests in tasks of subjective nature. For instance, Ferguson [17] observed conformity in tasks of attitudinal nature. Subsequently, Blake et al. [7] compared conformity effects across objective and subjective tasks. The authors note that higher conformity was observed in subjective tasks in comparison to objective tasks, as a result of higher normative influences in physical groups. They highlight that the motivation to achieve correct answers may have outweighed the appeal of conforming to an incorrect majority in objective questions. However, further work is required to understand whether and how objective and subjective questions may differ in eliciting conformity behaviour in online settings.

2.2.3 Self-confidence. Several studies have also investigated the effect of participant confidence on conformity behaviour $[11,61]$. Their results in unison emphasise a negative relationship between self-confidence and conformity $[11,61]$. This notion is in line with Deutsch and Gerard's view of informational influences, which suggests that individuals conform to the majority seeking the 'correct' response in uncertain situations [16]. However, the impact of self-confidence on social conformity is yet to be understood in online group settings.

2.2.4 Social Presence. Social presence has been described in the literature as the the awareness of and the sense of being connected to others via mediated communication [58]. It has also been defined as the ability of individuals to project themselves socially and emotionally as 'real' people in mediated communication [19, 23]. Furthermore, Short et al. [58] explained that social presence facilitated by a medium depends on its ability to convey the presence of the communicators through verbal and non-verbal cues. Despite the early perception that CMC is impersonal due to the absence of social context cues, results of subsequent studies contradicted this notion [23, 24, 62]. These studies further emphasised that online social presence is a complex, multifaceted concept which manifests itself across multiple dimensions (e.g., social context cues, interactivity and privacy), each with its own variables (e.g., interactivity could be measured in terms of timely responses, communication style, formality of language etc.) [24, 62].

Subsequently, a study exploring the impact of different communication mediums (e.g., face-to-face, telephone, chat, and email) on perceived social presence and interpersonal perceptions, identified that people are more likely to behave in a manner to be liked by others in a richer communication medium eliciting higher social presence [15]. More recent literature has built on this notion to understand the impact of different aspects of online social presence (such as user representation, interactivity, and response visibility) on social conformity. For example, previous work has observed that online user representations (a social context cue) with more anthropomorphic (human-like) features encouraged higher social attractiveness and trustworthiness of online interaction partners, leading to higher

, Vol. 1, No. 1, Article . Publication date: October 2021 
conformity in online group settings $[22,37]$. This behaviour was further explained in work by Nowak and Biocca [47], describing how the agency (the perception of communicating with a computer agent or a human being) and anthropomorphism offered by online user representations could significantly influence users' perceptions of social presence. Researchers highlight that participants reported higher social presence when representations were more realistic, human-like (high anthropomorphic) and perceived to represent a real human being. However, the online user representations used in the above studies were stereotypically gendered (which may also pose a significant impact on online conformity behaviour [35, 36, 67]) and are outdated (e.g., [37] compared text boxes against, stick figures and animated characters). Thus, further work is needed to explore the generalisability of these observations to more modern user representations.

Interactivity is another major dimension of online social presence [62], and has been investigated with regard to online social conformity. For example, Laporte et al. [33] compared conformity behaviour in participants answering an online quiz under two conditions: in a unidirectional setting where participants could see the answers of others alongside their profile pictures (no interaction), and in a bidirectional setting where participants were connected through a live video chat, capable of freely communicating with each other. They note higher conformity among participants in the live video condition, confirming the notion that individuals are more likely to conform in online settings with higher interactivity. Moreover, literature also supports the notion that online discussions could elicit both normative and informational influences leading to changes in an individual's opinions [49]

Furthermore, the impact of response visibility (i.e., whether user responses are visible to others or not) has been an interest within conformity research. The literature notes that conformity is considerably higher when users are informed that their responses are visible to the group (public) in comparison to situations where user responses are private [37]. Deutsch and Gerard [16] explained that being aware of others in public conditions leads to participants being susceptible to normative influences, in addition to the informational influences observed in private conditions.

While the impact of user representation, interactivity, and response visibility on online social conformity has been investigated independently, existing work does not account for their interaction effects, despite their likelihood to manifest simultaneously in realistic online settings. Thus, further work is required to investigate the impact of their interaction effects on online conformity. Moreover, it is possible that the effect of social presence on online conformity also depends on other well-known determinants such as majority group size, task objectivity and self-confidence, which is yet to be thoroughly investigated. Thus, in this study we aim to systematically examine how the aforementioned aspects of social presence impact online conformity behaviour in both objective and subjective tasks, with different majority - minority group compositions, while also accounting for effects of self-confidence.

\section{METHOD}

To investigate the effect of social presence on conformity behaviour in an online setting, we manipulate the level of social presence in terms of user representation (generic vs. user-specific avatars), interactivity (discussion vs. no discussion), and response visibility (public vs. private responses). To control these variables of online social presence, while simulating a plausible online group setting where users are required to make judgements, we deployed an online multiple choice questions (MCQ) quiz containing both subjective and objective questions. MCQ quizzes are frequently used in online social conformity experiments $[6,33,53,67,68]$ as they enable the simulation of a clear group majority and a minority while placing the participant in both these groups.

\subsection{The Quiz}

The online quiz contained 18 multiple choice questions ( 9 objective and 9 subjective questions). The objective questions were extracted from both existing literature [67] and popular online question repositories such as Sporcle and Britannica, which have been previously used in $[67,68]$ to extract quiz

, Vol. 1, No. 1, Article . Publication date: October 2021 


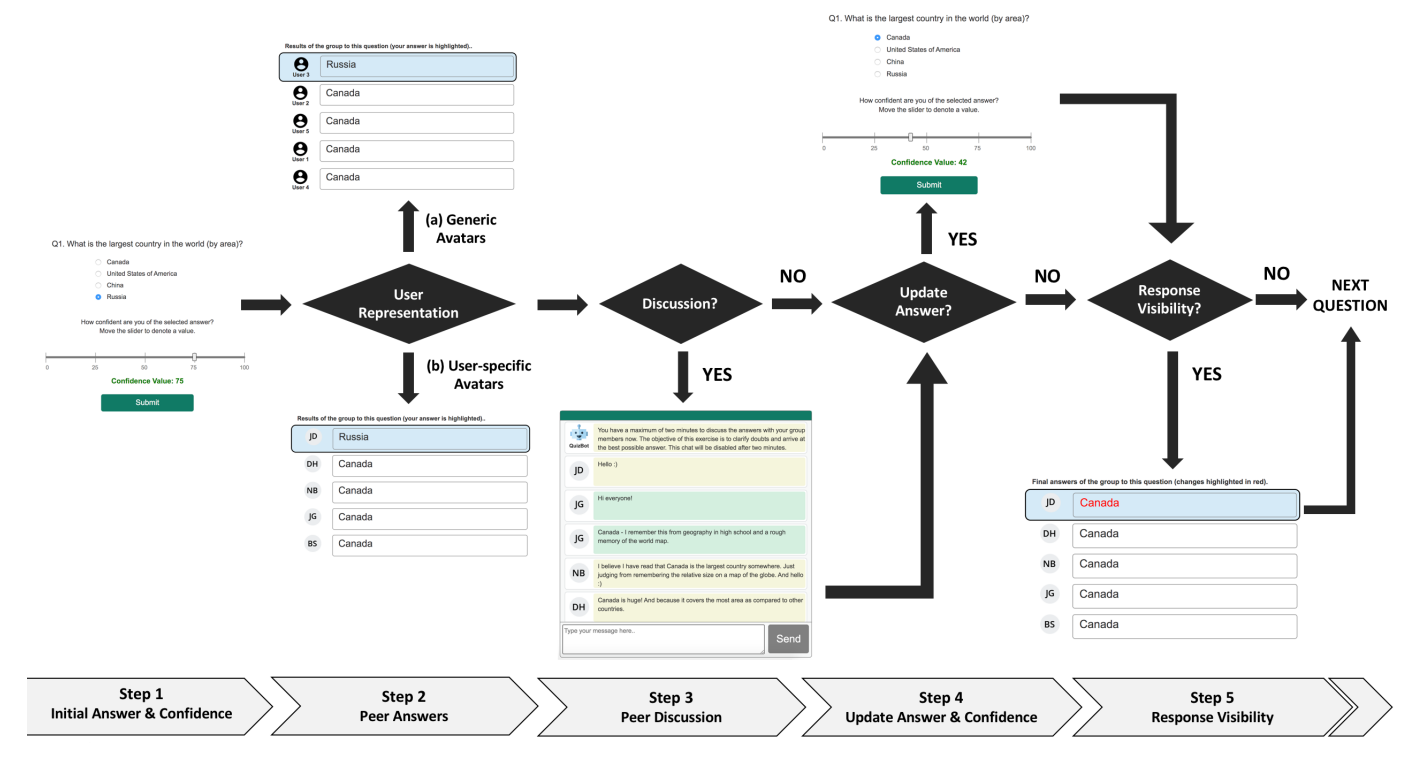

Fig. 1. Steps followed during the quiz by participants. Step 1: Initial answer and confidence, Step 2: Peer answers, Step 3: Peer discussion, Step 4: Update answer and confidence, Step 5: Response visibility

questions. This ensured that questions were only included if they were based on topics considered as general knowledge within the community considered for this experiment. Previous work investigating online social conformity through quizzes have also utilised general knowledge topics in their experiments $[33,53,67,68]$. The subjective questions were extracted from an article in ThoughtCo. outlining debating topics for high school students. We ensured that no overly sensitive topics were discussed in the quiz. A complete list of questions utilised in the quiz is included as supplementary material.

Participants followed the structure illustrated in Fig. 1 to complete the quiz. First, the participant is instructed to select their personal answer and rate their confidence on the chosen answer as illustrated in Step 1 of Fig. 1. Self-reported confidence levels were denoted using a scale ranging from $0-100$ with higher values representing higher levels of confidence. Subsequent to submitting their initial answer and confidence, the participant is shown a fabricated list of peer answers as chosen by four other participants completing the quiz (along with either generic or user-specific avatars as shown in Step 2(a) and Step 2(b) in Fig. 1). The fabricated peer answers were dynamically generated by our software to show the distribution of votes from other participants across a clear majority and a minority, while placing the participant in either group. The participant's answer was highlighted for convenience. This notion of using fabricated peer answers to investigate online social conformity was motivated by recent literature on conformity $[53,67,68]$. The next step determined the level of interactivity (discussion vs. no discussion) facilitated during the study. Participants in discussion conditions were given two minutes to discuss the group answers and their justifications with 'peers' through a real-time and text-based group chat as shown in Step 3 of Fig. 1. In reality, confederates of the researchers were used to simulate 'peer' discussions based on a predetermined script. Subsequently, all users were given the opportunity to change their answer option and/or confidence to finalise their answer (Step 4). Next, the response visibility (i.e., whether or not the final answers are visible to the rest of the group) was manipulated as shown in Step 5 of Fig. 1. Participants in public conditions were shown the final answers of the group with changed answers highlighted in red. After viewing the group's final answers, the user is taken to the next question. 


\subsection{Group Composition}

We chose an overall group size of five users (i.e., the size of the majority and the minority sums up to five) to investigate the effect of different majority - minority group distributions as illustrated in Fig. 2. While participants were informed that they will be connected with four peers to complete the quiz, there was only one real participant completing the quiz at any given time. Previous work investigating social conformity in both face-to-face and online groups has employed a similar group size $[7,16,30,37]$.

During the quiz, group answers were fabricated such that the participant was evenly placed in the majority (see group compositions (b) and (c) in Fig. 2) and in the minority group (see group compositions (d) and (e) in Fig. 2). We also simulated group consensus (in two questions) to provide a sense of authenticity to the peer answers. Moreover, we ensured that each group combination was equally tested against topics of both objective and subjective nature.

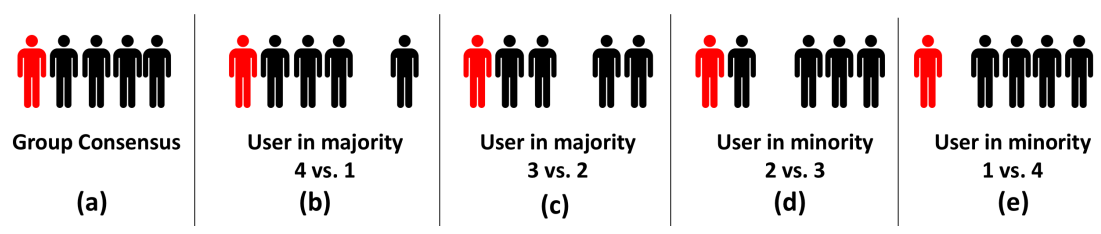

Fig. 2. Overview of group compositions investigated in the quiz (participant highlighted in red).

\subsection{Social Presence}

The main objective of this study was to investigate the compound effects of online social presence amidst other well-known determinants of conformity. Thus, we manipulated online social presence using three variables - user representation (generic vs. user-specific avatars), interactivity (discussion vs. no discussion) and response visibility (public vs. private responses) - which resulted in a $2 \times 2 \times$ 2 experimental design (8 conditions). Participants of each experimental condition interacted with a unique combination of the aforementioned interface elements. For example, participants assigned to the generic $\mathrm{x}$ discussion $\mathrm{x}$ public condition were represented using a single generic avatar, discussed answers with peers via a group chat, and saw final group answers, whereas participants in the user-specific $\mathrm{x}$ no discussion $\mathrm{x}$ private condition were denoted using user-specific avatars and did not see a group chat or final group answers. We now describe these experimental conditions in detail.

3.3.1 User Representation. User representations are important social context cues contributing to the perceived social presence in online settings [62]. In this study we investigate the impact of two commonly used online user representations: generic and user-specific avatars. In generic avatar conditions, a commonly used gender-neutral avatar is assigned to all the five users along with generic usernames such as "User 1" and "User 2" to differentiate between participants (see Step 2(a) in Fig. 1). Alternatively, in the user-specific avatar conditions, users are assigned dynamically generated avatars including the first letters of their first and last names (e.g., John Doe is represented by JD as shown in Step 2(b) in Fig. 1).

We highlight that our choice of user representations is based on literature explaining how agency and anthropomorphism associated with different online user representations could impact social presence in virtual group settings [22, 37, 47]. Based on the evidence provided in literature we hypothesise that user-specific avatars with user initials convey a stronger sense of being connected to a 'real' human being, than a single generic avatar with computer generated usernames. Moreover, the practice of assigning a default generic avatar to users is common in online social networks such as Twitter and YouTube and even in Learning Management Systems such as SAP Litmos and Docebo, where user decisions are likely to be influenced by others. Alternatively, some popular online 
platforms such as Google use user-specific avatars with user initials to represent them. The user representations used in this study are purposefully devoid of explicit user cues (such as gender, name, or age) to avoid confounding effects on online social conformity [12, 32, 35, 36, 39, 67].

3.3.2 Interactivity. We consider two levels of interactivity in this study: discussion and no discussion. This determines whether participants are given an opportunity to engage in a discussion with peers after viewing their answers (i.e., discussion) or not (i.e., no discussion).

In discussion conditions, users are informed that after being shown the peer answers, they will be given two minutes to discuss their answers and rationale behind selecting it, with others in the group as shown in Step 3 of Fig. 1. However, as we recruited only one participant per session, four confederates of the researchers pretended to be users participating in the quiz, to simulate a group discussion. The confederates engaged in the group discussion based on a script, dynamically created by the software for each question based on the fabricated answer distribution. Confederates have been used to simulate real users in previous work investigating social conformity in both face-toface $[7,16,30]$ and online [33] group settings. Alternatively, in no discussion conditions, participants are only shown the fabricated answer list of the group members. They are not subsequently given an opportunity to engage in a group discussion, and are directly taken to Step 4 of Fig. 1.

3.3.3 Response Visibility. After the peer answers are shown, participants are given the opportunity to update their answers and/or confidence prior to submitting their final answers (see Step 4 of Fig. 1). Response visibility determines whether the final answers are visible to the group (i.e., public) or not (i.e., private). Participants in the public conditions are informed prior to the quiz that their final answers will be visible to the group. Therefore, they are shown the list of updated answers of the group before moving to the next question, with updated answers highlighted in red as shown in Step 5 of Fig. 1. Moreover, to ensure that participants in public conditions are not suspicious of the authenticity of the answers, in $50 \%$ of the questions where at least one other simulated participant was placed in the minority with or without the user (e.g., see (b), (c) and (d) in Fig. 2), the said simulated participant changed their answer to that of the majority.

Alternatively, participants in private conditions are told that their final answers will not be visible to others in their group, and are taken to the next question upon submitting their final answers.

\subsection{Participants and Procedure}

We recruited 64 participants ( 32 men and 32 women) from different educational backgrounds which included engineering, science, marketing, management, arts and architecture fields. All participants were between 19 - 36 years of age and were recruited through our university's online notice board. Participants were equally distributed among the 8 experimental conditions with an equal number of men and women participating in each condition.

The study was conducted in a laboratory with one participant per session, under the supervision of a researcher. Participants were informed that they would be taking part in an online quiz together with four other participants. As the true purpose of the study could not be disclosed prior to the quiz as expected in studies investigating conformity behaviour [59], we explained that the study was motivated by the increasing use of online learning platforms and that we intend to investigate the performance of students in online quizzes. No other information triggering a competing or cooperative relationship between the participants was provided.

Before starting the quiz, participants completed an online form which collected their self-disclosed gender, age, and educational background. Upon submitting their demographic details, participants were randomly assigned to an experimental condition. The steps followed by the participant, and the interface they interacted with, depended on the condition they were assigned to as illustrated in Fig. 1. For instance, only participants assigned to discussion conditions, were shown an online group chat area and were greeted by a conversational agent named 'QuizBot'. This chat area was not visible to participants in no discussion conditions. Alternatively, participants in no discussion conditions

, Vol. 1, No. 1, Article . Publication date: October 2021. 
were given textual, step-by-step instructions through the study software (with no involvement of the researchers) prior to initiating the quiz.

The 'QuizBot' was utilised in discussion conditions to regulate group discussions (see Fig. 3) without the involvement of a researcher. The absence of a researcher closely replicates realistic online group settings, while also reducing the Hawthorne effect, a crucial aspect in conformity research $[1,66]$ The bot also signalled the participants when to start the discussion after viewing the peer answers and displayed the remaining time left for discussion, to ensure that group discussions were restricted to the allocated time frame of two minutes per question. The group chat was automatically disabled by the bot after the allocated time, and the users were prompted to the next step. Furthermore, the 'QuizBot' provided confederates regular updates on what the participant was doing before and after the discussion (i.e., answering the question, changing the answer after the discussion etc.), displayed the fabricated list of peer answers, and provided them prompts of the discussion points based on a predetermined script to minimise confusion and error.

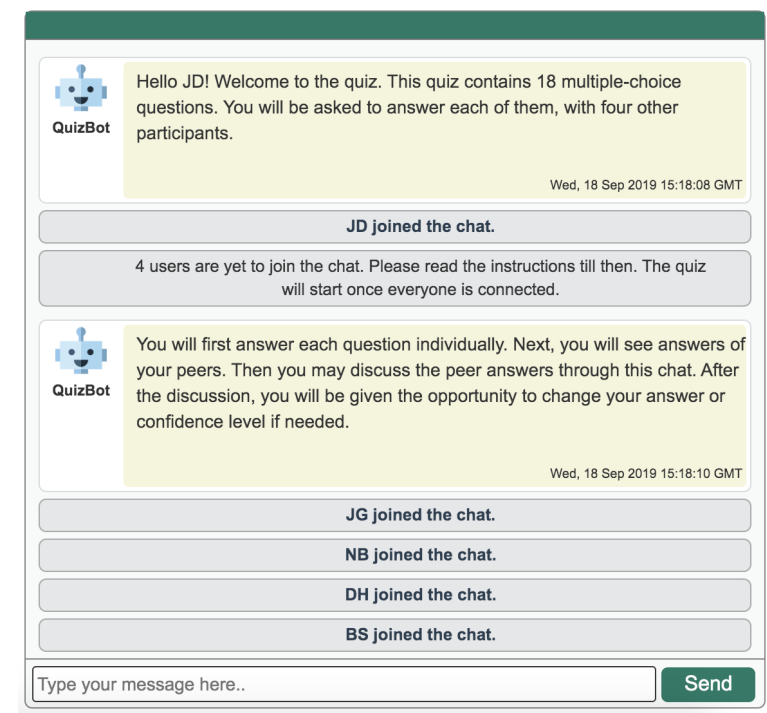

Fig. 3. QuizBot providing initial quiz instructions and informing participant status in the discussion conditions.

Upon completion of the quiz, participants participated in a brief semi-structured interview. The interview was arranged as follows. First, participants were asked for general thoughts on their experience participating in the study, to identify if they were suspicious of the authenticity of other participants. Subsequently, we inquired whether they changed their answers during the quiz and what factors contributed towards such behaviour. We were also interested in whether they were more inclined to change their answers in certain types of questions, to understand how task objectivity may affect their behaviour. Participants were also questioned about the impact of discussion, response visibility and user representations they were exposed to, to determine whether and how these three aspects of online social presence affected their conformity behaviour. Subsequently, we debriefed our participants on the true objective of the study. Participants were then given the opportunity to withdraw their participation and data collected during the study, if desired. All our participants consented to the use of the data collected, even upon revealing the study's true objective.

The experimental design was approved by the Ethics Committee of our university. The experiment lasted for approximately $60-90$ minutes per participant depending on the experimental condition they were assigned to, including briefing, completing the quiz, and the final interview. Each participant received a $\$ 20$ gift voucher for participation. 


\subsection{Preliminary Study}

Prior to the experiment, we conducted a preliminary study with 10 men and 10 women, where participants were asked to answer the same set of MCQs along with justifications for their answers. We obtained a total of 360 responses from the preliminary study. Next, we arranged the four answer options in each MCQ based on a descending order of the number of votes they received during the preliminary study, to determine the most popularly chosen answers for each question. This order was later considered along with the answers chosen by the participants in the main experiment, to dynamically determine the positioning of the majority and the minority groups when fabricating peer answers. For instance, in the question shown in Fig. 1 ("What is the largest country in the world (by area)?"), "Russia" and "Canada" were the top two answers chosen by the preliminary study participants. Thus, when a participant in the main study selected "Russia" as their initial answer, our software dynamically fabricated the peer answers placing "Canada" in a clear majority. The same approach was used to decide the minority's answer, when participants were placed in the majority. This ensured that the majority or the minority was always placed in a plausible answer option, regardless of being correct or incorrect.

Moreover, the justifications provided by participants in the preliminary study were used to create a script which was used by confederates to support their answers during the main experiment (in discussion conditions). This ensured that all justifications provided by the confederates simulating participants, closely represented how the considered community perceived the topic in question. This was especially crucial in the subjective questions. For objective questions, we chose justifications that could be used with all four of the answer options (e.g., "Canada/Russia/China/USA is huge! I also remember this fact from my geography class in high school."), and were counterbalanced among the four options during the experiment. We ensured that the justifications used in the final script did not include any obvious personal cues (such as gender or age) which could result in confounding effects on social conformity $[12,32,55]$. None of these explanations were explicit on the confidence level of the 'participant', or displayed uncertainty in the chosen answer. For instance, in the question shown in Fig. 1 the explanations provided by confederates could be as follows:

Confederate 1: "I remember from the world map that Canada is the biggest country in the world." Confederate 2: "Canada is huge! And because it covers the most area as compared to other countries."

Confederate 3: "I have read about this. Also by judging from remembering the relative size of Canada on a map of the globe."

Confederate 3: "I remember this as Canada from geography in high school and a rough memory of the world map."

\section{RESULTS}

All 64 participants answered 18 questions each, which resulted in a total of 1152 responses. Of these, participants were placed in the minority in 512 responses and in the majority or in a consensus in 640 responses (equally distributed among objective and subjective questions), to avoid drawing suspicion to the plausibility of the fabricated peer answers. On that note, we emphasise that our intention was not to compare results between majority and minority responses, but rather to investigate the impact of social presence on conformity across a diverse range of group compositions.

Upon displaying the fabricated peer feedback, participants were given the opportunity to:

- Change both initial answer option and confidence level.

- Change only their initial answer option.

- Change only their initial confidence level.

- Make no change to their initial answer.

Participants changed their initial answer and/or confidence at least once during the quiz, resulting in a total 431 changed responses with an average of 6.7 changes $(S D=4.1)$ per participant. Fig. 4 illustrates the distribution of the participants' post-feedback responses, when their answer was supported by 
a minority, or by a majority during the quiz. Participants were more inclined to change one's answer (with or without a change in confidence) when placed in a minority (in approximately $34 \%$ of the minority responses). On the other hand, being placed in the group majority was more likely to result in an increase in participant's self-confidence on selected answer (in approximately $28 \%$ responses). Thus, these results establish that participants changed their answers post feedback, not randomly but due to the influence of the predictors we considered, confirming the validity of our results.

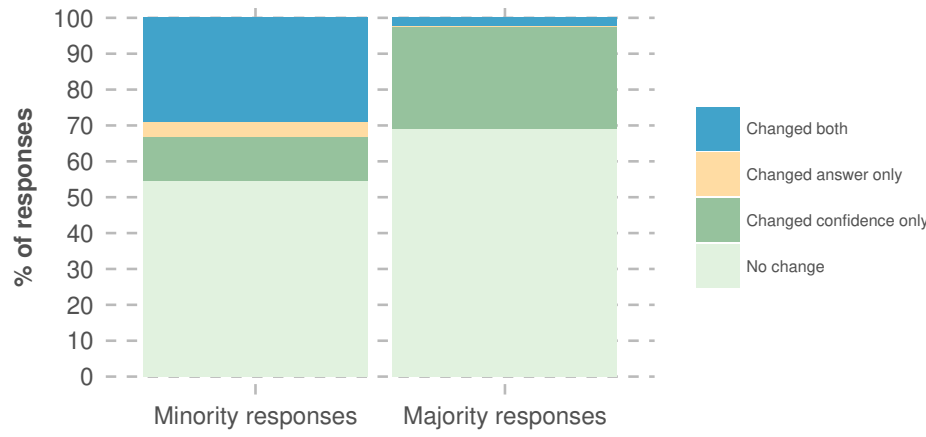

Fig. 4. Distribution of minority and majority responses across the four post-feedback response types.

\subsection{Model Construction}

For the purpose of this study, we define conformity as the act of changing one's answer to that of the majority. Our results show that 55 participants conformed at least once during the quiz, resulting in 152 conformity responses (approximately 30\% conformity), with an average of 2.4 conformity responses $(S \mathrm{D}=1.6)$ per participant. We then investigated the impact of the following ten predictor variables on the conformity behaviour of our participants. For our model construction, we only consider the responses of participants when placed in a minority, as the dependent variable was determining conformity behaviour.

- Majority size: Size of the majority (possible values: 3 or 4).

- Minority size: Size of the minority (possible values: 2 or 1 ).

- Group size difference: Difference between the majority group size and the minority group size (possible values : 1 or 3 ).

- User representation: The avatar used to represent users in the online platform (possible values: generic or user-specific).

- Interactivity: Whether or not users were given an opportunity to discuss peer answers with the group (possible values: discussion or no discussion).

- Response visibility: Whether or not the final group answers were visible to others (possible values: public or private).

- Task objectivity: Subjective or objective nature of the question.

- Initial confidence: Participant's confidence in answer prior to revealing peer answers (ranging from 0 to 100).

- Gender: Participant's self-disclosed gender.

- User ID: An unique identifier assigned to a given user during the quiz.

We used the R package lme4 [5] to perform a generalised linear mixed-effects model (GLMM) analysis of the relationship between the aforementioned variables and participant conformity. A GLMM allows us to identify the effect of a set of predictor variables on an outcome variable (conformity) while following an arbitrary (i.e., possibly non-normal) distribution. We specified participant (User ID) as a random effect to account for individual differences in our model. 
All statistically significant predictors included in the final model (following model selection through incremental removal of variables based on their predictive power) are shown in Table 1 . We perform a likelihood ratio test with the null model [8] and find that our model is statistically significant $\left(\chi^{2}=188.98, \mathrm{p}<0.001\right)$ and explains $41.8 \%$ of the variance in accuracy $\left(\mathbb{R}=0.65, \mathbb{R}^{2}=0.42\right)$. To ensure the validity of the model, we check for the existence of multicollinearity. Our predictors report a variance inflation factor between 1.05 and 1.46, well below the often-used threshold of 5 to detect multicollinearity [25].

\begin{tabular}{lll}
\hline Predictor & Coefficient & P-value \\
\hline Task objectivity (objective) & 1.92 & $<0.001$ \\
Group size difference & 0.69 & $<0.001$ \\
Initial confidence & -0.04 & $<0.001$ \\
No discussion : private & -1.15 & 0.017 \\
No discussion : public & -1.13 & 0.017 \\
Discussion : private & -0.97 & 0.039 \\
\hline
\end{tabular}

Table 1. Effect of statistically significant predictors on participant conformity. The sign of the coefficient (+/-) denotes the direction of the relationship between the predictor and conformity behaviour. Absolute value of the coefficient determines the effect size.

We observe statistically significant main effects of task objectivity (see Fig. 5), group size difference (see Fig. 6), and initial confidence of participants (see Fig. 7), while the level of interactivity (discussion vs. no discussion) and response visibility (public vs. private) demonstrate a statistically significant interaction effect on conformity behaviour (see Fig. 8). No effect was observed for participant gender or user representations used in this experiment. Next, we present a more detailed look at the significant features.

\subsection{Task Objectivity, Group Size Difference and Initial Confidence}

Our results show that the task objectivity (objective or subjective nature of the task) had the strongest effect on conformity behaviour. Out of the 152 conformity responses, 120 responses were related to objective questions $(79 \%)$ while only 32 responses were related to subjective questions $(21 \%)$, suggesting that participants were more inclined to conform to the majority's answer in objective questions than in subjective questions. We illustrate the likelihood of participants conforming to the majority's answers in subjective and objective questions in Fig. 5. We note that in subjective questions, participant conformity ranged between $0-50 \%$ with a median value of $0 \%$, while in objective questions the value ranged between $0-100 \%$ with a higher median value of $50 \%$.

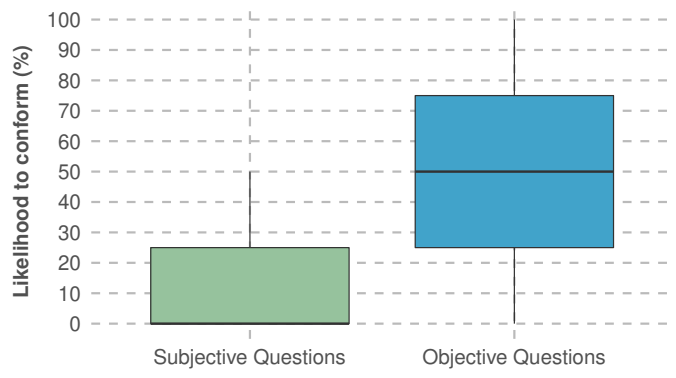

Fig. 5. The likelihood of participants conforming to the majority in subjective and objective questions. 
Moreover, the group size difference between the majority and the minority displayed a statistically significant main effect on conformity behaviour. We illustrate this relationship in Fig. 6 as a density plot, which shows the distribution of participants' likelihood of conforming, when the group size difference between the majority and the minority was 1 (majority of 3 vs. minority of 2) and 3 (majority of 4 vs. minority of 1). We note that the curve representing conformity behaviour when the group size difference is at 1, is left-skewed (with a mean of $24 \%$ and a median of $25 \%$ ), whereas the curve representing conformity behaviour when the group size difference is at 3 , is right-skewed (with a mean of $35 \%$ and a median of $25 \%$ ), suggesting that participants are more likely to conform to the majority when the difference between the groups is higher.

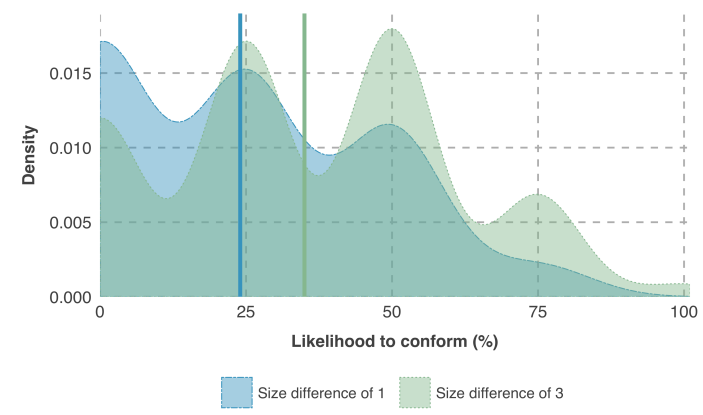

Fig. 6. The distribution of the likelihood of participants conforming to the majority against the considered group size differences. The solid vertical lines denote the mean likelihood to conform in each case.

Furthermore, our results show that individuals who display higher confidence on their initial answers are less likely to be influenced by the majority as demonstrated by the distribution of initial confidence levels of participants across non-conforming and conforming responses in Fig. 7. While the initial confidence level of participants ranges between $0-100$ in both non-conforming and conforming responses, the median initial confidence values is at 80 and 55 respectively.

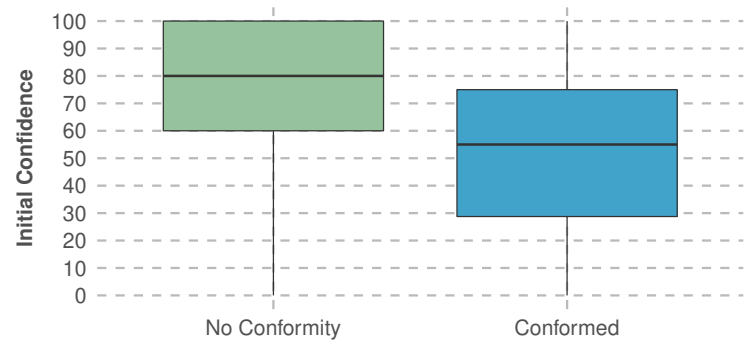

Fig. 7. Distribution of initial confidence values of participants across non-conforming and conforming responses.

\subsection{Social Presence}

We manipulated the social presence facilitated by the platform using three variables: user representation, interactivity and response visibility. While we do not find statistically significant main effects for each aspect, we note a statistically significant interaction between the level of interactivity (discussion vs. no discussion) and response visibility (public vs. private). The interaction effect between interactivity and response visibility results in four levels of social presence: discussion:public, discussion:private, no discussion:public, and no discussion:private. The effect of these four levels of social presence on conformity behaviour is illustrated in Fig. 8. 


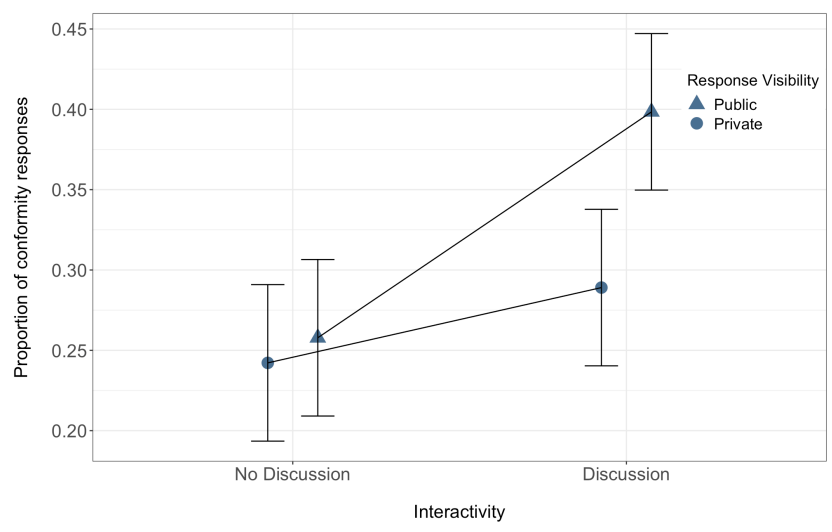

Fig. 8. Proportion of conformity responses across the four levels of interactivity and response visibility.

We highlight that highest conformity is observed when participants are provided the opportunity to discuss answers with peers, while also displaying their final answers to the rest of the group before moving to the next question (discussion:public condition). In contrast, participants are least likely to conform when there is no peer discussion, and the final responses are private. Moreover, based on our results in Table 1, we observe that even with private responses, having peer discussion is more likely to result in conformity when compared to conditions with no discussion, but with public responses.

\subsection{Qualitative Analysis}

Based on the transcripts from our semi-structured interviews, we aim to obtain a richer understanding of the quantitative results presented in the previous section. Two of the paper's authors individually transcribed and categorised the interview data, the outcomes of which were subsequently combined in an online spreadsheet to aid in the discussion and comparison of the categorisations. The two authors then collaboratively followed a deductive thematic analysis of the participants' responses [9]. In particular, our semi-structured interview aimed to identify the factors which participants believed to affect their shift in answer choices and confidence. We group our qualitative analysis across the manipulations concerning online social presence (user representation, interactivity, and response visibility) and factors previously highlighted in the (offline conformity) literature (task objectivity, majority group size, and self confidence).

4.4.1 Online Social Presence and Conformity. We altered the user representation of our participants (and their peers) using either generic avatars or user-specific avatars (using initials) and subsequently asked our participants how they perceived their assigned representation condition in contrast to the alternative. A number of participants mentioned that the use of initials created a more 'human-like' experience; "I like the initials better. It is more human and it kind of acknowledges each of us differently. We all have our own uniqueness, when compared to the generic avatars." (P17). Some participants highlighted how they subconsciously viewed user-specific avatars as 'real' people facing circumstances similar to them, which resulted in a connection to these participants; "I think in the discussion, when I saw avatars with initials, it put a person behind it for me rather than seeing "User 1" or "User 2". To me that would seem a little more automated than having avatars with initials. I think in a glimpse, I connected more with others and sometimes felt like I could relate to a particular user." (P64). Also, participants described how the use of initials supported in recognition of peers' (perceived) abilities to answer correctly; "If you have 20 - 30 questions, then we might see who is giving more correct answers consistently, with the avatars with initials." (P20). 
However, a widely shared perception among participants is that the level of representation is limited in both conditions. As described by $\mathrm{P} 01$, an individual's full name would reveal additional information as opposed to solely using initials; "If I see the full name and not just the initials, we can kind of guess the background of that person and where they are from. From there, you can guess what their experience and opinions might be.". Participants also describe how additional information not included in our study may be used to judge peers; "Neither option shows you their age, or experience and there is no way to know that person's background." (P32). In addition to the aforementioned factors of background and age, one participant suggested that the inclusion of academic titles would sway their judgement; "But if they have a title like "Professor", I will be more convinced." (P16).

We also asked our participants on the perceived effect of interactivity with their peers. We describe three distinct behaviours as observed in the interviews. First, participants note that chat-based interaction allows them to establish a level of confidence in the ability of their peers; "If I get a chance to talk with others, I will be more confident. I can determine whether they have the knowledge required. For example, if someone tells me 'I have been there before' to the capital of Bulgaria question, I will trust them. Else I will chose the majority." (P16). Second, participants note that the discussion allows them to obtain insights into the reasoning behind their peers' answers. This was particularly mentioned in relation to subjective questions. For example, "For most of the questions I was very firm about my answers. [...] But for subjective questions, I am happy to know why they chose their answers and then maybe change mine accordingly." (P01). Third, a small number of participants note that a group of participants could reach new insights through discussion; "Discussion will help people get new ideas as well." (P06).

However, we also note that a substantial portion of participants expressed that the discussions did not change their opinion in subjective questions. They emphasised how they have already made up their mind, and could only be swayed on objective questions if novel factual information would be shared; "I don't think that a discussion would make a change in subjective questions. I feel that I am entitled to have my opinion and they are entitled to theirs. Ijustified it pretty well in my head. For objective questions however, if I got new information from the discussion that would have made a difference." (P25).

When considering the effect of response visibility, a large portion of participants from the 'public' condition expressed concerns about the perception of their peers; "I don't want others to find me foolish. [...] I knew I was more likely to be wrong and I did not want myself to stand out and feel stupid." (P12). Participants which reported to be more at ease with the public visibility of their responses frequently cited the fact that the study was anonymous - thereby reducing the potential feeling of embarrassment; "I won't care about what others think whether I change or not. It is still anonymous, I don't think it has an effect on me." (P16). Participants did not only consider whether their peers would label them to be 'correct' or 'incorrect', but additionally considered how they were perceived. As illustrated by P46, "I might be a little pressured if they can see the changes. I would feel like they would think 'I was not good enough persuading User 3' so that adds a little pressure, because I am considering others' feelings. I don't want them to feel bad that they could not persuade me". Similarly, a participant assigned to the 'private' condition noted that even if he would not change his answer, he would use alternative signals to communicate with his peers (i.e., answer confidence); "If the amended answers were shown to others, I may reduce my confidence. Because others have different opinions and secondly, I would think others think "he is very stubborn, he won't change his answer'. I think this would negatively affect my personality." (P14).

4.4.2 Traditional Factors and Conformity. Task Objectivity (either objective or subjective in nature) had a significant effect on participants' conformity behaviour, with participants more likely to conform on objective questions. This sentiment was also observed in our interview data, with the majority of participants describing their reluctance to switch on subjective questions. The fact that they could not be 'wrong' on subjective questions was a widespread belief among participants; "Because it is all about my feelings. There is no correct or wrong answer." (P24). Furthermore, participants note how the distinction between peer and self-expertise is more critical when considering objective

, Vol. 1, No. 1, Article . Publication date: October 2021. 
questions; "If it was about opinions, usually I won't change because I have my own personal values about things. If it was more about intelligence, I will listen to others because I am not an expert in this area." (P16)

A number of participants reported that a more in-depth conversation would have the possibility to sway their mind on subjective questions, whereas an opposing majority on objective questions was sufficient motivation for switching to the majority opinion; "For subjective questions you need to read more about it. But for objective questions you know there is a correct answer and someone else can know it. While I wanted to know more about why others were thinking what they were thinking, two or three lines are not enough to change my opinion." (P42).

Group size differences, the size of the majority in comparison to that of the minority, were reported as having a considerable impact on participant responses. Participants were more confident in accepting answers from larger majorities than smaller majorities; "I often followed the majority because the majority may have the right answer. As we were a group of five, I was more sure of following the four person majority than a three person majority." (P18). If confronted with a unanimous majority of opposing answers, participants report conforming to the majority despite being confident in their own answer; "Even when I was pretty sure, there were some times where I changed my answer. It was because everyone else in the group chose something else." (P07).

Lastly, participants' initial confidence in their answer showed to be a significant predictor of their subsequent conforming behaviour. Our interview data confirms that participants were aware of this behaviour; "IfI had a lower confidence in the answer, I was more likely to change" (P10). Participants were unable to see the confidence of their 'peers', but this did not deter them from changing their answer; "Yes - Mainly when it was objective questions and in the case I had no idea of the answer, I often followed the majority. [...] Even if I did not know their level of confidence I still followed the majority." (P18).

\section{DISCUSSION}

As people continue to utilise online platforms to pursue social connections and support $[4,31,48$, $56,57]$, their experiences are susceptible to social conformity influences as observed in physical settings $[6,43,44,56]$. Interestingly, the literature suggests that online conformity has both positive and negative implications. For instance, conformity is not desired when individuals accept incorrect group judgements over personal decisions [6,29]. Biased social information leads to frequent errors, which could even nullify the perceived benefits of collective intelligence. However, existing work also highlights the importance of social conformity in strengthening online group relationships and creating a sense of belonging [56,57]. Moreover, conformity is also seen as a means to encourage acceptable group norms and standards within online communities, improving the quality of their output [60] Thus, it is imperative to understand what determines conformity behaviour in online settings in order to understand how future platforms can be designed to benefit from social conformity influences.

Our results show that previously established determinants of offline social conformity such as task objectivity, group size difference, and self-confidence pose significant effects on online conformity as well. We observe higher conformity in objective questions than in subjective questions, which contradicts the face-to-face literature on this regard [7]. Participants explained that conforming to the supposedly 'correct' answers of the majority in objective questions was more appealing than conforming to the majority's opinions in subjective questions to be 'liked' with a group. This implies that they are more susceptible to informational influences (the need to be 'right') than normative influences (the need to be 'liked') in online settings, possibly due to the anonymity and reduced social presence in online groups [16]. Furthermore, participants were more likely to conform as the distance between the majority and the minority increased, confirming the existing literature on majority group size in both offline and online contexts [3, 20, 30, 42, 54, 65, 67, 68]. Participants explained that larger distances between themselves and the majority exerted a sense of isolation and pressure to fit in, suggesting the existence of normative influences [16]. Moreover, we also note that participants were less pressured to conform when they were more confident about their initial responses, extending previous findings of face-to-face conformity literature $[11,61]$ to online settings. 


\subsection{Impact of Social Presence on Online Social Conformity}

Social presence is an important aspect of online interactions as it determines the degree to which we feel connected with our online correspondents $[58,62]$. While recent studies signify the importance of social presence on online user behaviour [18, 26-28, 64], its effect on conformity is underexplored. Moreover, despite being recognised as a multifaceted concept, existing work does not investigate multiple aspects of social presence simultaneously. Thus, in this study we investigate how social presence impacts online conformity behaviour across different user representations (generic vs. user-specific avatars), levels of interactivity (discussion vs. no discussion), and response visibility (public vs. private responses).

Our results show an overall conformity rate of $30 \%$, slightly lower than the conformity rates reported in face-to-face literature (usually above 33\% [2,3]). Differences in perceived social presence in face-to-face and online settings may have been a contributing factor in this regard, as highlighted by our participants during the interviews. In addition, our results imply that online social presence itself has a clear effect on conformity behaviour. While no main effects were observed for the three variables of social presence, higher social presence manipulated through interactivity (peer discussions) and response visibility (public responses) resulted in higher conformity. Our qualitative results confirm that participants in discussion conditions with public responses felt compelled to conform to the group answers, as their final answers were visible to peers subsequent to the discussion. Moreover, they were also concerned with how others would perceive their non-conforming behaviour despite the group's effort to convince them, suggesting susceptibility to normative influences. Thus, in comparison to recent work in online conformity highlighting the predominant effects of informational influences [67, 68], our study suggests the presence of both normative and informational influences, likely due to the added social presence via discussion and public responses.

Furthermore, we note that peer discussion itself without public responses (i.e., discussion:private) yielded more conformity than public responses in the absence of discussion (i.e., no discussion:public), suggesting that the level of interactivity imposed a higher contribution towards the perceived social presence than response visibility. Participants reported that discussion (high interactivity) provided them with an opportunity to understand the reasoning behind peer decisions, potentially increasing their confidence in the peer answers, resulting in increased conformity. Participants also highlighted how the discussion was most convincing when they could compare and relate their experiences with peer arguments, demonstrating high levels of social presence [41, 62].

We further note that the chosen user representations (generic vs. user-specific avatars) did not significantly differ in their influence on conformity. While, some participants highlighted during interviews that user-specific avatars were more effective in indicating the presence of a human user in comparison to generic avatars, the absence of explicit user cues (such as name, gender, age, etc.) may have invalidated this difference. Participants further suggested that real photographs or full names of their peers would have been more influential alternatives, as they provide more information about their peers and their background, which could have impacted their final decision.

\subsection{Implications for the Design of Online Group Settings}

Social presence is a crucial element of online platform design as it is seen to contribute towards platform attractiveness [26], user involvement and interaction [18, 26], user satisfaction [24, 52], and trust [27, 28]. Moreover, findings from our study acknowledge that social presence may also play a vital role in online conformity behaviour. We note that social presence could manifest in other means in online settings (e.g., communication style and strategy [62]), a topic beyond the scope of this paper. Thus, we present the following implications based on how online social presence can be manipulated in terms of interactivity, response visibility, and user representation.

We note that perceived social presence can be controlled via the level of interactivity and response visibility provided by an online setting, such that enhancing interactivity and response visibility leads to higher social presence and vice versa. Higher social presence would also increase the susceptibility

, Vol. 1, No. 1, Article . Publication date: October 2021 
of users to social influences such as conformity. Therefore, when higher conformity is desired (e.g., to encourage group norms and standards in online communities [56,60]), increasing the level of interactivity and visibility of user input is recommended. This would be particularly effective when normative conformity is desired (e.g., online support groups [56]).

While increasing perceived social presence in an online setting may seem desirable in most situations, we cannot disregard its effect on overall conformity behaviour. For example, the effect of social presence in online learning platforms has been debated in existing literature. While some studies suggest that social presence (in terms of peer interactions and feedback) leads to higher student satisfaction in online learning environments [23,24,52], other work shows that students who interact with peers are more likely to conform to erroneous judgements of their peers [6]. Therefore, platform designers should be aware of this conundrum when determining an appropriate balance between social presence and social influence.

Furthermore, our findings suggest that the visibility of user actions to others in an online setting is an important determinant of social presence as well as their conformity behaviour. When participants were told that their final answers would be visible to their peers, they were more inclined to adjust their answers in favour of the majority, as they were more concerned with being 'liked' than being 'right'. Thus, response visibility may be particularly important when it is desired to enhance normative conformity. This may be applicable to online support groups where co-dependency and group togetherness is more important compared to other online settings [56,57].

While our results do not demonstrate a clear difference in the impact on conformity between the generic and user-specific avatars, we highlight that participants preferred user representations with more information and human-like features, as suggested by [22, 37, 47]. However, we emphasise that while more information may improve the perceived social connection between users, having richer user representations (such as photographs or anthropomorphic avatars) may also generate stereotypical behaviour as seen in prior literature [35, 36, 50, 67]. For example, in [67] participants stereotypically perceived competency of others based on gender of their avatars, which in turn influenced their conformity behaviour. Thus, we recommend that future work investigate alternative online user representations devoid of explicit user cues (e.g., name, gender, age), such as default site-specific avatars as used in Slack and Snapchat, or animal avatars as used in Google Docs as shown in Fig. 9, to facilitate social presence without triggering similar stereotypical behaviour.
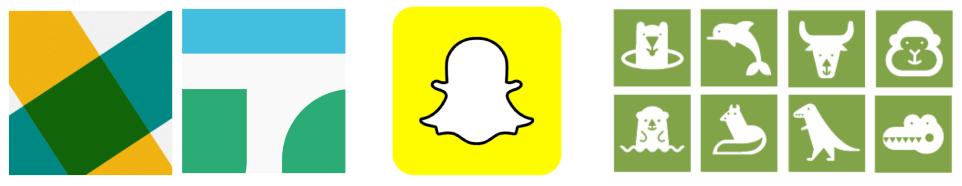

Fig. 9. Avatars used in Slack, Snapchat and Google Docs

In conclusion, our results acknowledge that social presence is a critical factor to be considered in online platform design. We emphasise that designers should pay special attention to how the level of interactivity, response visibility, and user representation could be used to control the perceived online social presence, thus also manipulating how susceptible users are to conformity influences. Future work could extend this work to investigate whether and how different platform designs could be developed, manipulating proven determinants of online social conformity (such as social presence, task objectivity, group size, self-confidence etc.) to encourage or discourage conformity and other social influences as required, to capitalise on their potential positive and negative implications. 


\subsection{Limitations}

We note the following limitations in our study. While our participants came from different educational backgrounds and levels, they demonstrated above average computer literacy. Thus, further work may be required to ensure whether these observations can be generalised to a wider population.

While within the community considered for this study (Australia), the chosen topics were acceptable as general knowledge questions (as supported by prior literature [67, 68], and our quantitative and qualitative results), they may not generate similar results cross-culturally. Different cultural backgrounds may in fact restrict the applicability of the current findings. Therefore, we encourage future research to extend our work by investigating different communities following a similar pilot test (as explained in Section 3.5) to ensure that chosen topics are acceptable as general knowledge in the targeted community.

Moreover, for the purpose of this experiment we defined social presence in terms of three aspects: user representation, interactivity, and response visibility. However, we note that social presence is a broader concept with other dimensions (e.g., social context, online communication [62]), which we did not consider in this study to avoid overly complicating the experimental setup. In addition, the aspects we did consider can also manifest in other ways. For example, interactivity can be determined via the communication style and formality of language in addition to what was tested in this study [62]. Therefore, we note that our study provides an initial step for future work that could further investigate other dimensions and aspects of social presence in online environments.

\section{CONCLUSION}

Recent literature on conformity has given a significant emphasis to its diverse manifestations in online settings [6, 43, 44, 56, 60, 67-69]. However, limited effort has been invested in understanding what triggers conformity in online settings. Therefore, this study investigated the effect of social presence (i.e., the awareness of and the sense of being connected to others via mediated communication [58]) on online conformity, while also accounting for the effects of majority group size, task objectivity, and self-confidence - well-known determinants of offline conformity. We manipulated the social presence facilitated by our study setup across three variables - user representation, interactivity, and response visibility - to investigate their combined effect on social conformity.

Our results reveal that in addition to the expected effects from group size difference, task objectivity, and self-confidence, the interaction among certain aspects of social presence also play a vital role on online conformity behaviour. We note that higher levels of online social presence (in terms of interactivity and response visibility) heightens the susceptibility of users to normative influences in addition to the commonly observed informational influences, leading to higher conformity in online settings.

We conclude with a discussion on what our findings imply for the design of online group settings. First, we emphasise that social presence can indeed be used to control conformity influences in online settings. Second, our results suggest that platform designers can manipulate the level of interactivity facilitated and the visibility of user actions to manage online social presence, which in turn could help regulate conformity influences. Thus, designers should pay special attention to the above aspects of social presence in order to encourage and shape user behaviour as desired in a given setting. We also highlight that the 'correct' level of social presence varies based on the requirement of the platform and thus should be determined after careful consideration of its pros and cons. Moreover, our findings emphasise that online users prefer user representations that can provide more user-specific information. However, as a substantial amount of literature investigating online user representations and stereotyping recommends otherwise [35, 36, 67], we suggest alternatives that provide a compromise between stronger user cues and stereotypical conformity. We encourage future work to investigate effects of other dimensions and variables of social presence, such as social context and communication style [62], on online conformity that can expand upon these implications. 


\section{REFERENCES}

[1] John G Adair. 1972. Demand characteristics or conformity?: Suspiciousness of deception and experimenter bias in conformity research. Canadian fournal of Behavioural Science/Revue canadienne des sciences du comportement 4, 3 (1972), 238.

[2] Solomon E Asch. 1951. Groups, leadership and men. Carnegie Press, Oxford, England, Chapter: Effects of group pressure upon the modification and distortion of judgements, 177-190.

[3] Solomon E Asch. 1955. Opinions and social pressure. Scientific American 193, 5 (1955), 31-35.

[4] Louise Barkhuus and Juliana Tashiro. 2010. Student Socialization in the Age of Facebook. In Proceedings of the SIGCHI Conference on Human Factors in Computing Systems (CHI '10). ACM, New York, NY, USA, 133-142. https://doi.org/10.1145/1753326.1753347

[5] Douglas Bates, Martin Mächler, Ben Bolker, and Steve Walker. 2015. Fitting Linear Mixed-Effects Models Using lme4. Journal of Statistical Software 67, 1 (2015), 1-48.

[6] Tanya Beran, Michelle Drefs, Alyshah Kaba, Noof Al Baz, and Nouf Al Harbi. 2015. Conformity of responses among graduate students in an online environment. The Internet and Higher Education 25 (2015), 63-69.

[7] Robert R Blake, Harry Helson, and Jane Srygley Mouton. 1957. The Generality of Conformity Behavior as a Function of Factual Anchorage. Difficulty of Task, and Amount of Social Pressure. Fournal of Personality 25, 3 (1957), 294-305.

[8] Benjamin M. Bolker, Mollie E. Brooks, Connie J. Clark, Shane W. Geange, John R. Poulsen, M. Henry H. Stevens, and Jada-Simone S. White. 2009. Generalized linear mixed models: a practical guide for ecology and evolution. Trends in Ecology \& Evolution 24, 3 (2009), 127-135.

[9] Virginia Braun and Victoria Clarke. 2006. Using thematic analysis in psychology. Qualitative Research in Psychology 3, 2 (2006), 77-101. https://doi.org/10.1191/1478088706qp063oa

[10] Michael J. Brzozowski, Phil Adams, and Ed H. Chi. 2015. Google+ Communities As Plazas and Topic Boards. In Proceedings of the 33rd Annual ACM Conference on Human Factors in Computing Systems (CHI '15). ACM, New York, NY, USA, 3779-3788. https://doi.org/10.1145/2702123.2702600

[11] Jennifer D Campbell, Abraham Tesser, and Patricia J Fairey. 1986. Conformity and attention to the stimulus: Some temporal and contextual dynamics. Journal of Personality and Social Psychology 51, 2 (1986), 315-324.

[12] Emily Christofides, Towhidul Islam, and Serge Desmarais. 2009. Gender stereotyping over instant messenger: The effects of gender and context. Computers in Human Behavior 25, 4 (2009), 897-901.

[13] Robert B Cialdini. 2001. Harnessing the science of persuasion. Harvard Business Review 79, 9 (2001), 72-81.

[14] Robert B Cialdini and Noah J Goldstein. 2004. Social influence: Compliance and conformity. Annu. Rev. Psychol. 55 (2004), 591-621.

[15] Joanie B Connell, Gerald A Mendelsohn, Richard W Robins, John Canny, and John Canny. 2001. Effects of communication medium on interpersonal perceptions. In Proceedings of the 2001 International ACM SIGGROUP Conference on Supporting Group Work. ACM, 117-124.

[16] Morton Deutsch and Harold B Gerard. 1955. A study of normative and informational social influences upon individual judgment. The fournal of Abnormal and Social Psychology 51, 3 (1955), 629-636.

[17] Leonard W Ferguson. 1944. An analysis of the generality of suggestibility to group opinion. Fournal of Personality 12, 3 (1944), 237-243.

[18] David R Fortin and Ruby Roy Dholakia. 2005. Interactivity and vividness effects on social presence and involvement with a web-based advertisement. Journal of business research 58, 3 (2005), 387-396.

[19] D Randy Garrison, Terry Anderson, and Walter Archer. 2001. Critical thinking, cognitive presence, and computer conferencing in distance education. American fournal of distance education 15, 1 (2001), 7-23.

[20] Solomon C Goldberg. 1954. Three situational determinants of conformity to social norms. The fournal of Abnormal and Social Psychology 49, 3 (1954), 325-329.

[21] Jorge Goncalves, Vassilis Kostakos, and Jayant Venkatanathan. 2013. Narrowcasting in Social Media: Effects and Perceptions. In Proceedings of the 2013 IEEE/ACM International Conference on Advances in Social Networks Analysis and Mining (ASONAM '13). ACM, New York, NY, USA, 502-509.

[22] Li Gong. 2008. How social is social responses to computers? The function of the degree of anthropomorphism in computer representations. Computers in Human Behavior 24, 4 (2008), 1494-1509.

[23] Charlotte N Gunawardena. 1995. Social presence theory and implications for interaction and collaborative learning in computer conferences. International journal of educational telecommunications 1, 2 (1995), 147-166.

[24] Charlotte N Gunawardena and Frank J Zittle. 1997. Social presence as a predictor of satisfaction within a computer-mediated conferencing environment. American journal of distance education 11, 3 (1997), 8-26.

[25] Joseph F Hair, William C Black, Barry J Babin, Rolph E Anderson, and RL Tatham. 2010. Multivariate Data Analysis. Pearson, New Jersey, NJ, USA.

[26] Khaled Hassanein and Milena Head. 2007. Manipulating perceived social presence through the web interface and its impact on attitude towards online shopping. International fournal of Human-Computer Studies 65, 8 (2007), 689-708.

[27] Khaled S Hassanein and M Head. 2004. Building online trust through socially rich web interfaces. In Proceedings of the 2nd annual conference on privacy, security and trust, Fredericton, New Brunswick, Canada. 15-22.

[28] Traci J Hess, Mark Fuller, and Damon E Campbell. 2009. Designing interfaces with social presence: Using vividness and extraversion to create social recommendation agents. Journal of the Association for Information Systems 10, 12 (2009), 1.

, Vol. 1, No. 1, Article . Publication date: October 2021. 
[29] Jessica Hullman, Eytan Adar, and Priti Shah. 2011. The Impact of Social Information on Visual Judgments. In Proceedings of the SIGCHI Conference on Human Factors in Computing Systems (CHI '11). ACM, New York, NY, USA, 1461-1470. https://doi.org/10.1145/1978942.1979157

[30] Chester A. Insko, Richard H. Smith, Mark D. Alicke, Joel Wade, and Sylvester Taylor. 1985. Conformity and Group Size: The Concern with Being Right and the Concern with Being Liked. Personality and Social Psychology Bulletin 11, 1(1985), 41-50.

[31] Adam N. Joinson. 2008. Looking at, Looking Up or Keeping Up with People?: Motives and Use of Facebook. In Proceedings of the SIGCHI Conference on Human Factors in Computing Systems (CHI '08). ACM, New York, NY, USA, 1027-1036. https://doi.org/10.1145/1357054.1357213

[32] Jitendra Kumar. 1983. Conformity behavior as a function of confederates' age and size of the confederate group Personality Study \& Group Behaviour 3 (1983), 69-73.

[33] Lieve Laporte, Christof van Nimwegen, and Alex J. Uyttendaele. 2010. Do People Say What They Think: Social Conformity Behavior in Varying Degrees of Online Social Presence. In Proceedings of the 6th Nordic Conference on Human-Computer Interaction: Extending Boundaries (NordiCHI '10). ACM, New York, NY, USA, 305-314.

[34] Bibb Latané. 1981. The psychology of social impact. American psychologist 36, 4 (1981), 343.

[35] Eun-Ju Lee. 2004. Effects of gendered character representation on person perception and informational social influence in computer-mediated communication. Computers in Human Behavior 20, 6 (2004), 779-799.

[36] Eun-Ju Lee. 2007. Wired for gender: Experientiality and gender-stereotyping in computer-mediated communication. Media Psychology 10, 2 (2007), 182-210.

[37] Eun-Ju Lee and Clifford Nass. 2002. Experimental tests of normative group influence and representation effects in computer-mediated communication: When interacting via computers differs from interacting with computers. Human Communication Research 28, 3 (2002), 349-381.

[38] John M Levine. 1999. Solomon Asch's legacy for group research. Personality and Social Psychology Review 3, 4 (1999), $358-364$

[39] Wendy Liu and Derek Ruths. 2013. What's in a name? Using first names as features for gender inference in twitter, In 2013 AAAI Spring Symposium Series. AAAI Spring Symposium - Technical Report SS-13-01, 10-16.

[40] Yong Liu, Jayant Venkatanathan, Jorge Goncalves, Evangelos Karapanos, and Vassilis Kostakos. 2014. Modeling What Friendship Patterns on Facebook Reveal About Personality and Social Capital. ACM Trans. Comput.-Hum. Interact. 21, 3, Article Article 17 (June 2014), 20 pages. https://doi.org/10.1145/2617572

[41] Patrick R Lowenthal and Chareen Snelson. 2017. In search of a better understanding of social presence: an investigation into how researchers define social presence. Distance Education 38, 2 (2017), 141-159.

[42] Paul Benjamin Lowry, Tom L Roberts, Nicholas C Romano Jr, Paul D Cheney, and Ross T Hightower. 2006. The impact of group size and social presence on small-group communication: Does computer-mediated communication make a difference? Small Group Research 37, 6 (2006), 631-661.

[43] Misa Maruyama, Scott P. Robertson, Sara Douglas, Roxanne Raine, and Bryan Semaan. 2017. Social Watching a Civic Broadcast: Understanding the Effects of Positive Feedback and Other Users' Opinions. In Proceedings of the 2017 ACM Conference on Computer Supported Cooperative Work and Social Computing (CSCW'17). ACM, New York, NY, USA, $794-807$.

[44] Misa T. Maruyama, Scott P. Robertson, Sara K. Douglas, Bryan C. Semaan, and Heather A. Faucett. 2014. Hybrid Media Consumption: How Tweeting During a Televised Political Debate Influences the Vote Decision. In Proceedings of the 17th ACM Conference on Computer Supported Cooperative Work \& Social Computing (CSCW'14). ACM, New York, NY, USA, 1422-1432.

[45] Katelyn YA McKenna and Amie S Green. 2002. Virtual group dynamics. Group Dynamics: Theory, Research, and Practice 6, 1 (2002), 116-127.

[46] Brian Mullen. 1983. Operationalizing the effect of the group on the individual: A self-attention perspective. Fournal of Experimental Social Psychology 19, 4 (1983), 295-322.

[47] Kristine L Nowak and Frank Biocca. 2003. The effect of the agency and anthropomorphism on users' sense of telepresence, copresence, and social presence in virtual environments. Presence: Teleoperators \& Virtual Environments 12, 5 (2003), 481-494.

[48] Julie Prescott, Terry Hanley, and Katalin Ujhelyi Gomez. 2019. Why do young people use online forums for mental health and emotional support? Benefits and challenges. British fournal of Guidance \& Counselling (2019), 1-11.

[49] Vincent Price, Lilach Nir, and Joseph N Cappella. 2006. Normative and informational influences in online political discussions. Communication Theory 16, 1 (2006), 47-74.

[50] Rabindra Ratan, David Beyea, Benjamin J Li, and Luis Graciano. 2019. Avatar characteristics induce users' behavioral conformity with small-to-medium effect sizes: a meta-analysis of the proteus effect. Media Psychology (2019), 1-25.

[51] Bernardo Reynolds, Jayant Venkatanathan, Jorge Gonçalves, and Vassilis Kostakos. 2011. Sharing Ephemeral Information in Online Social Networks: Privacy Perceptions and Behaviours. In Human-Computer Interaction - INTERACT 2011, Pedro Campos, Nicholas Graham, Joaquim Jorge, Nuno Nunes, Philippe Palanque, and Marco Winckler (Eds.). Springer Berlin Heidelberg, Berlin, Heidelberg, 204-215.

[52] Jennifer Richardson and Karen Swan. 2003. Examing social presence in online courses in relation to students' perceived learning and satisfaction. Journal of Asynchronous Learning 7, 1 (2003), 68-88. 
[53] Michael Rosander and Oskar Eriksson. 2012. Conformity on the Internet-The role of task difficulty and gender differences. Computers in Human Behavior 28, 5 (2012), 1587-1595.

[54] Leon Rosenberg. 1961. Group size, prior experience, and conformity. The fournal of Abnormal and Social Psychology 63, 2 (1961), 436-437.

[55] Victor Savicki, Merle Kelley, and E Oesterreich. 1999. Judgments of gender in computer-mediated communication. Computers in Human Behavior 15, 2 (1999), 185-194.

[56] Eva Sharma and Munmun De Choudhury. 2018. Mental Health Support and Its Relationship to Linguistic Accommodation in Online Communities. In Proceedings of the 2018 CHI Conference on Human Factors in Computing Systems (CHI '18) ACM, New York, NY, USA, Article 641, 13 pages.

[57] Eva Sharma and Munmun De Choudhury. 2018. Mental Health Support and Its Relationship to Linguistic Accommodation in Online Communities. In Proceedings of the 2018 CHI Conference on Human Factors in Computing Systems (CHI '18) ACM, New York, NY, USA, Article 641, 13 pages. https://doi.org/10.1145/3173574.3174215

[58] John Short, Ederyn Williams, and Bruce Christie. 1976. The social psychology of telecommunications. John Wiley \& Sons.

[59] David J Stang. 1976. Ineffective deception in conformity research: Some causes and consequences. European fournal of Social Psychology 6, 3 (1976), 353-367.

[60] Abhay Sukumaran, Stephanie Vezich, Melanie McHugh, and Clifford Nass. 2011. Normative Influences on Thoughtful Online Participation. In Proceedings of the SIGCHI Conference on Human Factors in Computing Systems (CHI '11). ACM, New York, NY, USA, 3401-3410.

[61] Abraham Tesser, Jennifer Campbell, and Susan Mickler. 1983. The role of social pressure, attention to the stimulus, and self-doubt in conformity. European fournal of Social Psychology 13, 3 (1983), 217-233.

[62] Chih-Hsiung Tu and Marina McIsaac. 2002. The relationship of social presence and interaction in online classes. The American journal of distance education 16, 3 (2002), 131-150.

[63] Jayant Venkatanathan, Evangelos Karapanos, Vassilis Kostakos, and Jorge Gonçalves. 2012. Network, Personality and Social Capital. In Proceedings of the 4th Annual ACM Web Science Conference (WebSci '12). Association for Computing Machinery, New York, NY, USA, 326-329. https://doi.org/10.1145/2380718.2380760

[64] Jayant Venkatanathan, Vassilis Kostakos, Evangelos Karapanos, and Jorge Gonçalves. 2013. Online Disclosure of Personally Identifiable Information with Strangers: Effects of Public and Private Sharing. Interacting with Computers 26, 6 (11 2013), 614-626. https://doi.org/10.1093/iwc/iwt058 arXiv:https://academic.oup.com/iwc/articlepdf/26/6/614/1962602/iwt058.pdf

[65] Eva Walther, Herbert Bless, Fritz Strack, Patsy Rackstraw, Doris Wagner, and Lioba Werth. 2002. Conformity effects in memory as a function of group size, dissenters and uncertainty. Applied Cognitive Psychology: The Official fournal of the Society for Applied Research in Memory and Cognition 16, 7 (2002), 793-810.

[66] Senuri Wijenayake, Niels van Berkel, and Jorge Goncalves. 2020. Bots for Research: Minimising the Experimenter Effect. In International Workshop on Detection and Design for Cognitive Biases in People and Computing Systems (CHI'20 Workshop). ACM, 9 pages.

[67] Senuri Wijenayake, Niels van Berkel, Vassilis Kostakos, and Jorge Goncalves. 2019. Measuring the Effects of Gender on Online Social Conformity. Proc. ACM Hum.-Comput. Interact. 3, CSCW, Article 145 (Nov. 2019), 24 pages. https://doi.org/10.1145/3359247

[68] Senuri Wijenayake, Niels van Berkel, Vassilis Kostakos, and Jorge Goncalves. 2020. Impact of contextual and personal determinants on online social conformity. Computers in Human Behavior (2020), 106302.

[69] Haiyi Zhu, Bernardo Huberman, and Yarun Luon. 2012. To Switch or Not to Switch: Understanding Social Influence in Online Choices. In Proceedings of the SIGCHI Conference on Human Factors in Computing Systems (CHI '12). ACM New York, NY, USA, 2257-2266. https://doi.org/10.1145/2207676.2208383 


\section{University Library}

\section{- M M N E R VA A gateway to Melbourne's research publications}

Minerva Access is the Institutional Repository of The University of Melbourne

Author/s:

Wijenayake, S;Van Berkel, N;Kostakos, V;Goncalves, J

Title:

Quantifying the Effect of Social Presence on Online Social Conformity

Date:

2020-05-28

Citation:

Wijenayake, S., Van Berkel, N., Kostakos, V. \& Goncalves, J. (2020). Quantifying the Effect of Social Presence on Online Social Conformity. Proceedings of the ACM on Human-Computer Interaction, 4 (CSCW1), pp.1-22. https://doi.org/10.1145/3392863.

Persistent Link:

http://hdl.handle.net/11343/272730 